\title{
PFAPA syndrome in the Czech Republic: a single- centre experience
}

\author{
Petra Król", Marek Böhm, Dana Nemcová, Pavla Doležalová \\ From 2011 Pediatric Rheumatology Symposium sponsored by the American College of Rheumatology \\ Miami, FL, USA. 2-5 June 2011
}

\begin{abstract}
Purpose
PFAPA syndrome (Periodic Fever, Aphthous stomatitis, Pharyngitis and Adenitis) is an idiopathic autoinflammatory disease with the first manifestation before 5 years of age. Fever attacks lasting for 3-6 days have individual periodicity within about 3-8 week intervals. They are accompanied by individual combination of other symptoms among which culture-negative pharyngitis/tonsillitis, oral aphthae and cervical adenitis are the most common. Children are healthy and thriving in between attacks. Clinical benign course with no long-term sequelae with normal growth and development is typical for PFAPA syndrome. Fever attacks usually do not regress with antibiotic therapy, but a single prednisone dose of $1 \mathrm{mg} / \mathrm{kg}$ administered at the onset of fever has a dramatic effect. Tonsillectomy appears a promising curative method for more difficult PFAPA patients.
\end{abstract}

\section{Aim}

To describe clinical and laboratory characteristics of a single-centre cohort of PFAPA patients.

\section{Methods}

Retrospective chart review (patients diagnosed 20042007) and prospective collection (2008-2010) of clinical and laboratory data during febrile attack and in afebrile interval.

\section{Results}

56 boys and 53 girls with PFAPA syndrome were diagnosed. Median age of the first manifestation was 20 months, median interval between attacks was 4 weeks and fever duration was 3,5 days. Patients were followed for median of 56,6 months. Fever was associated with pharyngitis/tonsillitis (87\%), cervical adenitis (73\%) and aphthous stomatitis (39\%). Laboratory measures during the fever attack (median, range) were as follows: CRP $(63,4 ; 12$ $237,4 \mathrm{mg} / \mathrm{l})$, ESR $(31 ; 9-60 / \mathrm{h})$, WBC (14; range 3-20x109/ 1). In all patients where follow-up values were available inflammatory parameters dropped back to normal within at least 2 weeks without fever. All patients had normal IgD levels and normal mevalonate in urine collected during the fever attack. A single prednisone dose was effective in about $90 \%$ of patients. Tonsillectomy lead to the complete remission in 12/13 children. Presence of self-limited recurrent fever with tonsillitis in early childhood of one of the parents was recorded in $45 \%$ of families.

\section{Conclusion}

PFAPA syndrome appears to be a relatively common cause of recurrent fever in early childhood in the Czech Republic. Detailed analysis of clinical and laboratory data including long-term outcomes is ongoing.

\section{Disclosure}

Petra Król: None; Marek Böhm: None; Dana Nemcová: None; Pavla Doležalová: None.

Published: 13 July 2012

doi:10.1186/1546-0096-10-S1-A85

Cite this article as: Król et al:: PFAPA syndrome in the Czech Republic: a

single-centre experience. Pediatric Rheumatology 2012 10(Suppl 1):A85.

General University Hospital in Prague, Praha 2, Czech Republic

(c) 2012 Król et al; licensee BioMed Central Ltd. This is an Open Access article distributed under the terms of the Creative Commons BHoWed Central Attribution License (http://creativecommons.org/licenses/by/2.0), which permits unrestricted use, distribution, and reproduction in any medium, provided the original work is properly cited. 